\title{
EFECTO DE LA DENSIDAD DE SIEMBRA EN EL CRECIMIENTO DE Piaractus brachypomus (Cuvier, 1818) “PACO” EN ESTANQUES SEMINATURALES DE PUCALLPA
}

\author{
Sonia Deza ${ }^{1}$, Socorro Quiroz ${ }^{2}$, Mariano Rebaza ${ }^{1}$, Carmela Rebaza $^{1}$
}

\section{RESUMEN}

El objetivo del presente trabajo fue determinar la densidad de siembra adecuada para Piaractus brachypomus, "paco". Se utilizó el diseño experimental de Bloques Completos al Azar utilizando una matriz $\mathrm{W}=3 \times 3$. Los tratamientos utilizados fueron T 1:5 000 peces ha $a^{-1}$ x 3, T 2:10 000 peces ha-1 ${ }^{-1}$ 3, T 3:15000 peces ha-1 x 3. Se sembraron un total de 744 alevinos de "paco" obtenidos por reproducción artificial con longitud y peso promedio inicial de $8.5 \mathrm{~cm}$ y $10.4 \mathrm{~g}$, respectivamente. El alimento utilizado fue balanceado con $33 \%$ de proteína bruta. La tasa de alimentación inicial y final fue del $10 \%$ y $2.5 \%$ de la biomasa, respectivamente. Los resultados obtenidos no muestran diferencias significativas en longitud, peso, tasa de crecimiento específico, factor de conversión de alimento, eficiencia alimenticia y factor de condición entre tratamientos. Al incrementar la densidad de siembra, el rendimiento $\left(\mathrm{k} \mathrm{ha}^{-1}\right)$ se incrementó significativamente.

Palabras clave: Densidades, crecimiento, paco, Piaractus brachypomus.

\section{ABSTRACT}

The objective of this research was to determine the appropriate culture density in Piaractus brachypomus "paco". The experimental design of randomized blocks with a W $=3 \times 3$ matrix, was used. The treatments were T:1: 5000 fish $\mathrm{ha}^{-1} \times 3, \mathrm{~T} 2: 10000$ fish $\mathrm{ha}^{-1}$ x 3, T 3: 15000 fish ha-1 x 3.744 alevines of "paco", obtained by artificial reproduction, with initial average longitude and weight of $8.5 \mathrm{~cm}$ and $10.4 \mathrm{~g}$, respectively, were used. The food was balanced with $33 \%$ of gross protein. The beginning and ending feeding rate was $10 \%$ to $2.5 \%$ of the biomass, respectively.

1 Biólogos pesqueros del Instituto de Investigaciones de la Amazonía Peruana- IIAP. Programa de Ecosistemas Acuáticos. Centro Regional de Investigaciones de Ucayali. km 12.4 de la Carretera Federico Basadre. Telefax: 061-573732. E-mail: iiapu@terra.com.pe. Pucallpa - Perú.

2 Tesista de la Universidad Nacional de Trujillo, Facultad de Ciencias Biológicas, San Martín 380. Telefax 044 235841-Aptdo 315. E-mail: biologic@chanchan.unitru.edu.pe. Trujillo - Perú. 
The obtained results do not show significant differences in longitude, weight, rate of specific growth, factor of food conversion, nutritive efficiency and condition factor among treatments. When increasing the density, the yield $\left(\mathrm{k} \mathrm{ha}^{-1}\right)$ increased significantly.

Key words: Density, growth, paco, Piaractus brachypomus.

\section{INTRODUCCIÓN}

La piscicultura es una actividad productiva relativamente reciente en la Amazonía peruana. Desde su inicio hasta hace unos cinco años se practicó en forma limitada, principalmente a nivel familiar, y durante los últimos años ha tenido un desarrollo acelerado, debido fundamentalmente a los avances obtenidos en el proceso de producción de alevinos de especies nativas como Colossoma macropomum "gamitana" y Piaractus brachypomus "paco" (Reyes, 1998). Desde entonces, el Instituto de Investigaciones de la Amazonía Peruana (IIAP) ha generado tecnologías para la crianza de especies como Colossoma macropomum "gamitana", Piaractus brachypomus "paco" y Prochilodus nigricans "boquichico". Estas especies han sido priorizadas teniendo en cuenta su rusticidad, adaptación al cultivo, excelente tasas de crecimiento y conversión alimenticia, y por aceptar muy bien el alimento balanceado, permitiendo preveer que estas especies cultivadas en estanques, serán en los próximos años la base de gran parte de la producción acuícola de la región Ucayali (Rebaza, et al., 1999).

En 1993, el Perú reportó la producción de pescado de especies amazónicas producidas en cautiverio, correspondiendo $58 \mathrm{tm}$ a gamitana, $20 \mathrm{tm}$ a paco y $10 \mathrm{tm}$ a boquichico. Estos niveles de producción actualmente se han incrementado, debido a que las técnicas de cultivo artesanal que se practicaban han experimentado una notable expansión y diversificación que tienden alcanzar el nivel comercial (Reyes, 1998). En la región Ucayali, se ha alcanzado para el año 1999 una producción total de $59074 \mathrm{tm}$ (DIRESEPE, 1999).

Trabajos relacionados a densidades de siembra en "paco" son escasos, Lovshin et al., (1974), realizaron estudios preliminares en esta especie, utilizando una densidad de 2632 peces ha $^{-1}$, obteniendo pesos promedios finales de $0.99 \mathrm{k}$ en 360 días de cultivo con una dieta de $29 \%$ de proteína; cuando incrementaron la densidad a 4400 peces ha $^{-1}$ y el nivel de proteína de la dieta a $35 \%$, el rendimiento que obtuvieron fue de $4605 \mathrm{k} \mathrm{ha}^{-1}$. Da Silva et al., (1978), encontraron mejores rendimientos para esta especie a una densidad de carga de 5000 peces ha $^{-1}$. Estevez (1995), utilizando una 
densidad de siembra de 0.15 peces $\mathrm{m}^{-2}$ obtuvo peces de $1 \mathrm{k}$ de peso en 174 días de cultivo, utilizando como alimento concentrado para pollos. Alcántara \& Padilla (1998), demostraron la ausencia de diferencias significativas entre densidades de $3000 \mathrm{y}$ 4000 peces ha' ${ }^{-1}$.

El objetivo del presente trabajo fue determinar el efecto de la densidad de siembra en el crecimiento de "paco" para lograr una alta producción de este importante recurso hidrobiológico.

\section{MATERIALES Y MÉTODOS}

\section{Lugar experimental}

El presente estudio fue realizado de abril a diciembre de 1999, en la estación experimental del IIAP-Ucayali, ubicado a la margen derecha de la Carretera Federico Basadre, a $12.4 \mathrm{~km}$ de la ciudad de Pucallpa, distrito de Yarinacocha, provincia de Coronel Portillo, región Ucayali. Geográficamente está ubicada a $8^{\circ} 22^{\prime} 31^{\prime}$ " de latitud sur y 74³4'35" de longitud oeste, a una altitud de $154 \mathrm{msnm}$. El clima es cálido húmedo, con temperaturas promedios anuales de $26^{\circ} \mathrm{C}$ y precipitación promedio de 1 $780 \mathrm{~mm}$ al año.

\section{Diseño experimental}

Se utilizó el diseño experimental de Bloques Completos al Azar (Arroyo, 1984), con tres tratamientos y tres repeticiones cada uno, correspondiendo a una matriz $\mathrm{W}=3 * 3$.

T $1: 5000$ peces ha-1 $\times 3$

T 2 : 10000 peces ha $^{-1} \times 3$

T 3 : 15000 peces ha $^{-1} \times 3$

\section{Variables evaluadas}

1. Longitud total $(\mathrm{cm})$

2. Peso (g)

3. Tasa de crecimiento específico $(\mathrm{G})$

$\mathrm{G}=\ln$ (peso final) $-\ln ($ peso inicial) $\times 100$ período (días)

(Ricker, 1975 citado por Silva et al, 1997) 
4. Factor de conversión de alimento (F.C.A.)

F.C.A. $=$ Alimento balanceado seco ofrecido

Peso húmedo ganado

(Martínez, 1987)

5. Eficiencia alimenticia (E.A.)

E.A. $=$ Peso húmedo ganado $\times 100$

Alimento seco ofrecido

(Martínez, 1987)

6. Factor de condición (K)

$\mathrm{K}=\mathrm{P} \times 100$

$\mathrm{L}^{3}$

(Lagler, 1996 y Ricker, 1971 citado por Martínez, 1987)

7. Tasa de sobrevivencia, $(\%)$

8. Rendimiento $\left(\mathrm{k} \mathrm{ha}^{-1}\right)$

9. Análisis económico de los costos de producción unitario por $\mathrm{k}$ de carne para cada uno de los tratamientos y del punto de equilibrio. Para el análisis de rentabilidad se consideró los indicadores económicos: TIR (Tasa Interna de Retorno) y VAN (Valor Actual Neto). Para el cálculo de estos indicadores se realizó un flujo de caja para cada uno de los tratamientos, considerando los siguientes supuestos:

- Considera un horizonte de cinco años, suponiendo que un productor puede trabajar durante cinco años sin necesidad de hacerle mejoras al estanque.

- La inversión en estanque y equipo se realiza en el año cero, es decir, antes de iniciar la operación.

- Los ingresos son producto de la venta del pescado y en cada uno de los cinco años va a tener el mismo rendimiento. También es considerado como ingreso, en el quinto año, el valor de la inversión del estanque menos lo que se ha cargado como depreciación en los cinco años de operación, es decir, se considera un valor de recuperación suponiendo que el productor después del quinto año puede vender su estanque a ese precio.

- Como egresos de dinero se suponen los costos de operación que se han calculado anualmente. 


\section{Infraestructura piscícola}

El experimento se desarrolló en 3 estanques seminaturales, de forma rectangular con profundidades promedios de 1 a $1.20 \mathrm{~m}$, utilizándose un área total de $744 \mathrm{~m}^{2} \mathrm{de}$ espejo de agua. Estos estanques fueron divididos en 9 unidades experimentales, los cuales, a su vez, fueron debidamente preparados, realizándose el vaciado total. Luego, fueron encalados con cal agrícola en una proporción de $1500 \mathrm{k} \mathrm{ha}^{-1}$ con la finalidad de corregir el pH del agua y además como medida profiláctica. El llenado se hizo con agua proveniente de un pozo tubular de $25 \mathrm{~m}$ de profundidad. Para el fertilizado se utilizó abono orgánico, de tipo gallinaza, en una proporción de $1500 \mathrm{k} \mathrm{ha}^{-1}$

\section{Material biológico}

Se trabajó con 744 alevinos de Piaractus brachypomus "paco" que fueron obtenidos por reproducción artificial en el IIAP-Ucayali, con longitudes y pesos promedios iniciales de $8.5 \mathrm{~cm}$ y $10.4 \mathrm{~g}$. Los registros biométricos se realizaron mensualmente. El tamaño de la muestra evaluada fue el $25 \%$ del total de la población sembrada por cada tratamiento. Los registros mensuales de incrementos de peso sirvieron para la corrección mensual de la tasa de alimentación.

\section{Alimentación de los peces}

Se utilizó una dieta balanceada peletizada con $33 \%$ de proteína bruta, elaborada con harina de pescado, torta de soya como fuentes de proteína, maíz molido y polvillo de arroz, como fuentes de energía. La frecuencia de alimentación fue diaria, y la tasa de alimentación inicial y final fue de $10 \%$ y $2.5 \%$, respectivamente.

\section{Control de la calidad del agua}

Se obtuvo información sobre la calidad del agua de las unidades experimentales, determinándose los parámetros físicos: temperatura ambiental y del agua, la transparencia; los parámetros químicos: oxígeno disuelto en $\mathrm{mg}^{\mathrm{l}^{-1}}$, anhídrido carbónico libre en $\mathrm{mg} \mathrm{l}^{-1}$ y total en meq $\mathrm{l}^{-1}$, alcalinidad en $\mathrm{mg} \mathrm{l}^{-1}$, dureza total en $\mathrm{mg}^{-1} \mathrm{y}$ finalmente el $\mathrm{pH}$ del agua. La metodología para los análisis químicos del agua se hizo según Fukushima et al., (1982). 
Durante la época de verano se realizaron recambios de 50\% del nivel de agua, después de cada muestreo.

\section{Procesamiento estadístico}

El nivel de significancia entre tratamientos se determinó aplicando el análisis de medias $(\mathrm{p}<0.05)$, a través de la prueba de Duncan. El análisis de varianza y de medias de las variables estudiadas fueron procesadas por el paquete estadístico S.A.S. (Science Analysis Statistics) .

\section{RESULTADOS Y DISCUSIÓN}

Los resultados obtenidos indicaron que en las variables de longitud y peso final alcanzados por los peces no mostraron diferencias significativas entre tratamientos a nivel de varianza, ni a nivel del comparativo de medias (Tabla 1). No obstante, el tratamiento 5000 peces ha-1 muestra un ligero incremento en comparación con los otros dos, y los pesos alcanzados a esta densidad corresponde a pesos comerciales que tienen gran aceptación en los mercados locales. Ferrari y Bernardino (1984), reportan iguales resultados con la especie Piaractus mesopotamicus, a densidades de 5000 y 10000 peces ha $^{-1}$ sin encontrar diferencias significativas en longitud y peso. Según los resultados observamos que el incremento de la densidad de siembra retarda el crecimiento en los peces, como consecuencia hay crecimiento lento. Al respecto Reyes (1998), indica que la densidad de siembra de los peces afecta el crecimiento de los peces en proporción inversa, es decir, que si se incrementa la densidad se reduce la tasa de crecimiento específico, entonces, los peces tardarán más tiempo en alcanzar el peso comercial.

Tabla 1. Longitud y peso promedio final de Piaractus brachypomus "paco" sembrados a tres densidades en 240 días de cultivo.

\begin{tabular}{llc}
\hline Tratamientos $\left({\left.\text { peces } x h a^{-1}\right)}^{2}\right.$ & Longitud total & Peso \\
\hline 5000 & $28.33 \mathrm{a}^{*}$ & $505.70 \mathrm{a}^{*}$ \\
10000 & $27.83 \mathrm{a}$ & $457.23 \mathrm{a}$ \\
15000 & $26.67 \mathrm{a}$ & $420.00 \mathrm{a}$ \\
C.V. & $3.65 \%$ & $10.57 \%$ \\
$\mathrm{R}^{2}$ & 0.97 & 0.94 \\
\hline
\end{tabular}

* Letras iguales no presentan diferencias significativas: Duncan $(\mathrm{p}<0.05)$ 
La tasa de crecimiento específico es mayor en el tratamiento 5000 peces ha ${ }^{-1}$ en comparación con los otros dos (Tabla 2). El tratamiento 15000 peces ha $^{-1}$ presentó una tasa de crecimiento específico por debajo de los otros $\left(1.54 \% \mathrm{~g} \mathrm{día}^{-1}\right)$. Del análisis de varianza y del comparativo de medias se obtuvo que no había diferencia significativa entre tratamientos. Estos resultados coinciden con los encontrados por Ferrari y Bernardino (1984), al trabajar con dos densidades de 5000 y 10000 peces ha $^{-1}$, no encontrándose diferencias significativas entre tratamientos.

El factor de conversión alimenticia y la eficiencia alimenticia no tuvieron diferencias significativas estadísticamente entre tratamientos (Tabla 3). El alimento utilizado para el género Piaractus en diferentes trabajos en América Latina, varían del 14\% al 40\% de proteína bruta, con una tasa de alimentación del 2 al $5 \%$ de su biomasa, alcanzando conversiones alimenticias de 1.9 a 3.8 (Bernardino \& Ferrari et al., 1989). Al trabajarse en el experimento con una dieta con $33 \%$ de proteína, una tasa de alimentación inicial y final de $10 \%$ y $2.5 \%$, se obtuvieron conversiones alimenticias de 0.54 a 3.55 , siendo el tratamiento 5000 peces ha $^{-1}$ el que alcanzó un valor de 1.09 , el más próximo a 1 en comparación con los otros dos. Halver (1972), afirma que es muy difícil obtener valores de conversión de alimento iguales o menores que 1, pero, cuando esto ocurre, se debe considerar la cantidad de alimento natural que puede ser capturado por los peces, ya que los organismos más pequeños que hay en el estanque, como los unicelulares y los rotíferos, son un buen alimento para los peces, especialmente en la fase alevinos y juveniles. Estos alimentos naturales abastecen con los nutrientes esenciales que los peces necesitan para alcanzar su máximo crecimiento potencial (CEIA, 1999; Woynarovich \& Woynarovich, 1998); es posible que esto explique los valores bajos de la conversión alimenticia.

Tabla 2. Parámetros de crecimiento para Piaractus brachypomus "paco" en tres densidades de siembra durante 240 días de cultivo.

\begin{tabular}{lccccc}
\hline Tratamientos & $\begin{array}{c}\mathrm{G}\left(\mathrm{g} \mathrm{día}{ }^{-1}\right) \\
(\%)\end{array}$ & F.C.A. & $\begin{array}{c}\text { E.A. } \\
(\%)\end{array}$ & K & $\begin{array}{c}\text { Sobrevivencia } \\
(\%)\end{array}$ \\
\hline 5000 peces ha $^{-1}$ & $1.62 \mathrm{a}^{*}$ & $1.09 \mathrm{a}^{*}$ & $92.90 \mathrm{a}^{*}$ & $2.19 \mathrm{a}^{*}$ & $96.9 \mathrm{a}^{*}$ \\
10000 peces ha $^{-1}$ & $1.58 \mathrm{a}$ & $1.26 \mathrm{a}$ & $81.03 \mathrm{a}$ & $2.07 \mathrm{a}$ & $91.9 \mathrm{a}$ \\
15000 peces ha $^{-1}$ & $1.54 \mathrm{a}$ & $1.33 \mathrm{a}$ & $77.26 \mathrm{a}$ & $2.21 \mathrm{a}$ & $89.5 \mathrm{a}$ \\
$\mathrm{CV}$ & $3.64 \%$ & $12.65 \%$ & $10.56 \%$ & $7.76 \%$ & \\
$\mathrm{R}^{2}$ & 0.90 & 0.76 & 0.80 & 0.44 & \\
\hline
\end{tabular}

$\mathrm{G}=$ Tasa de crecimiento específico $\quad$ F.C.A $=$ Factor de conversión alimenticia.

E.A.=Eficiencia alimenticia $\mathrm{K}=$ Factor de condición.

* Letras iguales no presentan diferencia significativa: Duncan $(p<0,05)$. 
Tabla 3. Análisis de varianza del rendimiento $\left(\mathrm{k} \mathrm{ha}^{-1}\right)$ en 240 días de cultivo.

\begin{tabular}{lccc}
\hline $\begin{array}{l}\text { Fuentes de } \\
\text { variabilidad }\end{array}$ & $\begin{array}{c}\text { Grados de } \\
\text { libertad }\end{array}$ & Cuadrado medio & Significancia \\
\hline Tratamientos & 2 & 721596.433 & $* *$ \\
Bloques & 2 & 6676786.9948 & $*$ \\
Error & 4 & 918638.793 & \\
Total & 8 & & \\
\hline
\end{tabular}

** Altamente significativo

* Significativo

Tabla 4. Rendimiento $\mathrm{k} \mathrm{ha}^{-1}$ de Piaractus brachypomus "paco" en tres densidades de siembra durante 240 días de cultivo.

\begin{tabular}{|c|c|}
\hline Tratamientos & Rendimiento kg ha-1 \\
\hline 5000 peces ha $^{-1}$ & $2413.53 \mathrm{a}$ \\
\hline 10000 peces $\mathrm{ha}^{-1}$ & $4080.31 \mathrm{a} \mathrm{b}$ \\
\hline 15000 peces $\mathrm{ha}^{-1}$ & $5592.30 \mathrm{~b}$ \\
\hline
\end{tabular}

Letras iguales no presentan diferencia significativa: Duncan $(\mathrm{p}<0.05)$

El factor de condición encontrado estuvo cercano al valor de 2, lo cual indica que la contextura del "paco" fue normal, así mismo, los valores superiores a 2 nos indican la gran capacidad y posibilidades de esta especie para desarrollar un sistema de cultivo intensivo (Martínez, 1987). En esta variable tampoco se encontró diferencias significativas entre tratamientos.

La sobrevivencia en general fue alta, resultando porcentajes de $96.9,91.9$ y $89.5 \%$ para los tres tratamientos, respectivamente. Estos resultados demuestran que el "paco" es un pez que se adapta a su ambiente y soporta densidades altas de cultivo.

En cuanto al rendimiento, se demostró que hay diferencia significativa entre tratamientos, siendo el tratamiento de 15000 peces $\mathrm{h}^{-1}$ superior a los otros dos, con rendimientos de $5592.3 \mathrm{k} \mathrm{ha}^{-1}$ en 240 días. Con las otras dos densidades, de 10000 y 5000 peces ha $^{-1}$, se obtuvieron rendimientos de 4080.3 y de $2413.5 \mathrm{k} \mathrm{ha}^{-1}$, respectivamente (Tabla 4). Al respecto CEIA (1999), indica que el rendimiento potencial máximo es más alto en aguas con mayor capacidad de carga por unidad, dado que el rendimiento aumenta, con un incremento en la densidad, cuando la tasa de crecimiento no es afectada por la población. 
Tabla 5. Costos de producción anual de Piaractus brachypomus "paco" en tres densidades de siembra.

\begin{tabular}{lrrr}
\hline Concepto & 5000 peces ha $^{-1}$ & 10000 peces ha $^{-1}$ & 15000 peces ha $^{-1}$ \\
\hline Costos fijos & & & \\
Depreciación infraestructura & 867 & 867 & 867 \\
Depreciación equipamiento & 439 & 439 & 439 \\
Costos variables & 7268 & 11682 & 15491 \\
Costo total & 8574 & 12988 & 16797 \\
Producción (k) & 2414 & 4080 & 5592 \\
Costo unitario & 3.55 & 3.18 & 3.00 \\
Costo variable unitario & 3.01 & 2.86 & 2.77 \\
Precio (S/.) & 5.00 & 5.00 & 5.00 \\
Punto de equilibrio (k) & 656.76 & 611.13 & 585.65 \\
\hline
\end{tabular}

Tabla 6. Análisis de rentabilidad para Piaractus brachypomus "paco" en tres densidades de siembra.

\begin{tabular}{cccc}
\hline Densidades & TIR (\%) & VAN & TD (\%)* \\
\hline 5000 peces ha $^{-1}$ & 13.48 & $(5.141)$ & 20 \\
10000 peces ha $^{-1}$ & 28.27 & 6.583 & 20 \\
15000 peces ha $^{-1}$ & 42.19 & 17.801 & 20 \\
\hline
\end{tabular}

(*) Tasa de descuento a la que se actualizó el VAN: Tasa de interés del financiamiento.

Al realizar el análisis económico, el costo unitario de producción es menor en la densidad de 15000 peces ha $^{-1}$ ya que se obtiene 2.77 soles por $\mathrm{k}$, mientras que en la densidad de 5000 peces ha $^{-1}$ el costo fue de 3.01 soles por $\mathrm{k}$ (Tabla 5). El tratamiento de 15000 peces ha $^{-1}$ fue el mejor, al obtenerse un TIR de $42.19 \%$, ya que resultó ser muy superior a la tasa de interés en el mercado (20\%). Este tratamiento parece ser la mejor alternativa ya que le dejaría al productor una utilidad de 17801 nuevos soles después de recuperar todos sus gastos de inversión y operativos (Tabla 6). Con esta experiencia quedan descartados los sistemas tradicionales de cultivo que se basan en la producción a una tasa de crecimiento máximo desde el punto de vista biológico y que, sin embargo, no son buenos cuando se determina la tasa de crecimiento económicamente óptima, lo cual tiene que ver con el costo de los insumos.

Por otro lado, se afirma que el crecimiento de los peces está influenciado en gran medida por las condiciones ambientales que prevalecen en el ecosistema cerrado, tales como la temperatura, oxígeno disuelto, $\mathrm{pH}, \mathrm{y}$ alimento disponible entre otros 
(Reyes, 1988). Consideramos importante analizar estos aspectos para relacionarlos con el comportamiento de crecimiento de la especie en estudio. En la Tabla 7 se describen los parámetros físico-químicos del agua de los estanques experimentales.

Los valores de temperatura registrados durante el experimento, estuvieron dentro del rango óptimo considerados por Díaz \& López (1989), quienes manifiestan que se obtienen mejores crecimientos con temperaturas entre 26 y $29^{\circ} \mathrm{C}$. Durante el desarrollo del cultivo se registraron temperaturas mínimas de $16.6{ }^{\circ} \mathrm{C}$ y máximas de $34.3{ }^{\circ} \mathrm{C}$, estos cambios bruscos de temperatura trajeron como consecuencia el crecimiento lento de los peces y la disminución de la tasa de crecimiento específico, en los meses en que hubo estos cambios de la temperatura del agua. Algunos autores como González \& Heredia (1989), Ferrari et al., (1990), Bernardino \& Ferrari (1989), Woynarovich A \& E. Woynarivich (1998), y Angelini \& Petrere (1992), afirman que las temperaturas altas o bajas pueden ocasionar estrés en los peces y reducen su crecimiento. Cuando las temperaturas bajan influyen en la alimentación, metabolismo y crecimiento de los peces, porque este parámetro está directamente ligado al consumo del alimento, esto posiblemente fue la causa de que el factor de conversión de alimento aumentara a 3.55 y la eficiencia alimenticia disminuyera a $30.43 \%$, sin consecuencias de mortalidad en los peces.

Tabla 7. Promedios mensuales de los factores físico-químicos del agua de los estanques experimentales.

\begin{tabular}{lcccccc}
\hline Meses & $\begin{array}{c}\text { Temperatura } \\
\text { Superficial } \\
\left({ }^{\circ} \mathrm{C}\right)\end{array}$ & $\begin{array}{c}\text { Oxígeno } \\
\text { Disuelto } \\
\left(\mathrm{mg} \mathrm{l}^{-1}\right)\end{array}$ & $\mathrm{pH}$ & $\begin{array}{c}\text { Anhídrido } \\
\text { carbónico } \\
\text { libre }\left(\mathrm{mg} \mathrm{l}^{-1}\right)\end{array}$ & $\begin{array}{c}\text { Anhídrido } \\
\text { carbónico total } \\
\left(\mathrm{meq} \mathrm{1}^{-1}\right)\end{array}$ & $\begin{array}{c}\text { Dureza } \\
\text { Total } \\
\left(\mathrm{mg} \mathrm{l}^{-1}\right)\end{array}$ \\
\hline Siembra & 27.30 & 5.5 & 7.52 & 9.00 & 0.51 & 76.67 \\
Mayo & 27.22 & 4.43 & 6.81 & 8.50 & 0.56 & 34.67 \\
Junio & 26.58 & 3.12 & 6.67 & 14.08 & 0.86 & 38.00 \\
Julio & 25.82 & 2.99 & 6.55 & 11.92 & 0.88 & 51.00 \\
Agosto & 25.71 & 1.04 & 6.22 & 28.17 & 0.86 & 33.000 \\
Setiembre & 27.97 & 1.74 & 6.45 & 23.75 & 0.80 & 21.33 \\
Octubre & 27.54 & 1.87 & 6.67 & 34.33 & 0.74 & 24.00 \\
Noviembre & 27.78 & 2.91 & 6.52 & 38.83 & 0.73 & 30.27 \\
Diciembre & 28.73 & 3.46 & 6.67 & 26.00 & 0.62 & 28.50 \\
\hline
\end{tabular}

En relación con el contenido de oxígeno disuelto del agua, algunos autores indican que la concentración de este parámetro debe mantenerse entre 3 y $6.5 \mathrm{mg} \mathrm{l}^{-1}$ (Díaz \& López, 1993), sin embargo, esta especie es muy resistente a aguas con bajas 
concentraciones de oxígeno ( 1 a $3 \mathrm{mg} \mathrm{l}^{-1}$ ), durante el experimento se encontraron valores mínimos hasta de $1.04 \mathrm{mg} \mathrm{l}^{-1}$, sin embargo, no causó mortalidad de los peces.

El pH debe encontrarse entre 6.5 y 8.5 , ya que un pH más ácido o más alcalino por un largo período de tiempo, disminuirá el crecimiento de los peces (Sipaúba, 1998). Así mismo, Boyd (1996), indica que el pH está ligado estrechamente con los niveles de $\mathrm{CO}_{2}$, observándose altos niveles de $\mathrm{CO}_{2}$ en tanto es bajo el $\mathrm{pH}$. El pH del agua de los estanques varió de 6.22 a 7.52, valores muy cercanos a lo que recomienda Sipaúba (1998).

Reyes (1998), menciona que en la zona de Pucallpa la dureza del agua de los estanques es baja, alcanzando valores de $16.34 \mathrm{mg} \mathrm{l}^{-1}$, siendo los valores registrados para este parámetro ligeramente superiores; esta fluctuación se ha presentado durante el período de lluvias y de sequía, llegando a $21.33 \mathrm{mg} \mathrm{l}^{-1}$ en setiembre y $51 \mathrm{mg} \mathrm{l}^{-1}$ en julio, lo cual evidencia que la cantidad de sales minerales disminuye por efecto de las lluvias.

Es importante tener en cuenta el control de la calidad de agua al decidir el sistema de cultivo a aplicarse. Díaz et al., (1993) indica que la densidad de siembra más recomendada en cultivos semi-intensivos para "cachamas" es un ejemplar $\mathrm{m}^{-2}$ y esta densidad se puede incrementar de 1.5 a 2 y 2.5 ejemplares para cultivos intensivos con gran exigencia en el control del recambio de agua, calidad del agua y en la alimentación artificial. Con los resultados obtenidos en el presente estudio, la densidad obtenida para "paco" puede incrementarse a 1.5 ejemplares $\mathrm{m}^{-2}$ teniendo en consideración los aspectos anteriormente señalados. La producción de carne de este recurso a través de la piscicultura permitirá cubrir en parte la demanda de este producto rico en proteínas y a precios al alcance del poblador.

\section{CONCLUSIONES Y RECOMENDACIONES}

El cultivo de "paco" a densidades de siembra de 5000 peces ha ${ }^{-1}, 10000$ peces ha $^{-1}$ y 15000 peces $^{-1} \mathrm{a}^{-1}$, no muestran diferencias significativas $(\mathrm{p}<0.05)$, en la longitud, peso, tasa de crecimiento específico, factor de conversión de alimento, eficiencia alimenticia y factor de condición. Sin embargo, en la densidad de 1.5 ejemplares $\mathrm{m}^{-}$ ${ }^{2}$ el rendimiento de "paco" se incrementó significativamente.

Se recomienda aplicar la densidad de siembra de 1.5 ejemplares $\mathrm{m}^{-2}$ al sistema de cultivo actual, teniendo en consideración la calidad de agua, recambios y la alimentación. 


\section{AGRADECIMIENTO}

Expresamos nuestro agradecimiento al economista Miguel Valdivieso por su apoyo en la elaboración del análisis económico del presente estudio.

\section{BIBLIOGRAFIA}

ANGELINI, R.; PETRERE Jr., M. 1992. Simulação da produção do "pacu” Piaractus mesopotamicus em viveiros da piscicultura. Boletín técnico. CEPTA, Pirassununga, V(5). P.41-55.

ALCÁNTARA F.; PADILLA, P. 1998. Rendimiento de Piaractus brachypomus y Colossoma macropomum criados en estanques a distintas densidades, IIAP. Nota científica. 5p.

ARROYO, R. 1984. Estadística aplicada a la investigación: Diseños experimentales. Instituto de investigaciones de la Amazonía Peruana. Iquitos. 160p.

BERNARDINO, G.; FERRARI, V. 1 989. Efeitos do uso de ração comercial no desempenho do pacu, Piaractus mesopotamicus (Holmberg 1 887) em cativeiro. Centro de Pesquisa e Treinamento em Acuicultura - CEPTA. Boletín Técnico. Vol (2).p. 1- 89.

BOYD, C. 1 996. Manejo de suelo y de la calidad de agua en la acuicultura de piscinas Asociación Americana de Soya.

CALZADA, J. 1 982. Métodos estadísticos para la investigación. Universidad Agraria La Molina. Edic.5ta. Edit. Milagros. Lima-Perú. P. 120-125.

CEIA. 1999. Curso sobre el desarrollo de la piscicultura. Centro Egipcio Internacional para la Agricultura. El Cairo-Egipto.

CEPTA. 1 987. Síntesis de trabajos realizados con especies del género Colossoma y Piaractus. Pirassununga. Brasil. 37p.

COCHRANE, T.; SÁNCHEZ, P. 1 982. Recursos de tierras, suelos y su manejo en la región amazónica: Informe acerca del estado del conocimiento. En: Amazonía Investigación sobre agricultura y uso de tierra. CIAT. Cali Colombia. p. 141-218. 
CÓRDOVA, P. 1 993. Alimentación animal. Concytec. Edit. Editee del Perú S.R. Ltda. Lima-Perú. 244p.

DA SILVA A.; CANEIRO, A.; MELO, F.; LOVSHIN, L. 1 978. Mono y policultivo intensivo de Colossoma macropomum y Piaractus brachypomus. México. $20 \mathrm{p}$.

DÍAZ G.; LÓPEZ, B. 1 993. El cultivo de la "cachama blanca" (Piaractus brachypomus) y de la "cachema negra" (Colossoma macropomum). En fundamentos de Acuicultura Continental. INPA. Colombia.

DIRECCIÓN REGIONAL SECTORIAL DE PESQUERIA-UCAYALI (DIRESEPE). 1 999. Informe técnico.

ESTEVEZ R. 1 995. La Cachama. Cultivo en estanques. INDERENA. Bogotá. Colombia. 30p.

FERRARI, V.; LUCAS A.; GASPAR, L. 1 990. Monocultura de pacu Piaractus mosopotamicus Holmberg, 1887 em condiçoes de viveiro-estufa. Centro de Pesquisa e Treinamento em Aquicultura. Boletín Técnico. Vol.(3). p. $1-63$

FUKUSHIMA, M; REYES, W.; SIFUENTES, G.; SHIMOKAWA, L.; SALDAÑA, G.; CASTTILLO G. 1 982. Manual de Métodos Limnológicos. Universidad Nacional de Trujillo. Trujillo-Perú. 250p.

GONZÁLES, J.; HEREDIA, B. 1 989. El cultivo de la "cachema” Fondo Nacional de Investigación Agropecuaria (FONAIAP). Estación Experimental de Guárico, sub estación Guanapito. Maracay. Venezuela. 124p.

HALVER, J. 1 972. Nutrición de peces. New York. 713p.

LOVISHING, L.; DA SILVA, A.; CANEIRO, A.; MELO, F.. 1 981. Biology and Culture Potential of Colossoma sp. Native to South America, Mimeo. En: Hibridación de paco, Piaractus brachuypomus (Cuvier, 1 818) por gamitana, Colossoma macropomum (Cuvier, 1 818) en Iquitos-Perú. Folia Amazónica Vol. (4) 1992.

LOVISHING L.; DA SILVA, A.; FERNÁNDEZ, J.; SOBRINO, A. (1 974). Cultivo de Piaractus brachypomus y Colossoma macropomum. Brasil. 24p. 
MARTÍNEZ, M. 1 987. Métodos de evaluación, control y racionamiento en la alimentación práctica. Alimentación en Acuicultura, Comisión Asesora de Investigación Científica y Técnica. Espinoza y Labarta editores. Madrid España. P. 295-325.

REBAZA, C.; DEZA, S.; VILLANUEVA, C.; OLIVA, R. 1 999. Diagnóstico técnico económico de la piscicultura en las provincias de Coronel Portillo y Padre Abad de la Región Ucayali. Pucallpa-Perú 43p.

REYES, W. 1 998. Cultivo de peces amazónicos. Revista Peruana de Limnología y Acuicultura Continental. Publicación especial APLAC. N 4. Trujillo-Perú.

SILVA, J.; BERNARDINO, G.; SILVA, M.; FERRARI, V.; MENDOCA, J. 1997. Cultivo do "pacú" Piaractus mesopotamicus (Holberg, 1 818) em duas densidade do estocagem no Nordeste do Brasil. Centro Nacional de Pesquisa de Peixes Tropicais (CEPTA). Boletín técnico. Vol (10). P. 1-70.

SIPAÚBA, L. 1 988. Limnología aplicada a aquicultura. Unidad Complementaria de la Universidad Estatal Paulista. Centro de Aquicultura. Boletim Técnico $\mathrm{N}^{\mathrm{o}}$ 1. Brasil.

WOYNAROVICH, A.; WOYNAROVICH, E. 1 998. Reproducción artificial de las especies Colossoma y Piaractus. Guía detallada para la producción de alevinos de gamitana, paco y caraña. FONDEPES. Taller. Lima-Perú. 


\section{ANEXOS}

Tabla 1. Flujo de caja para el tratamiento de 5000 peces $\mathrm{x} \mathrm{ha}^{-1}$

\begin{tabular}{rrrrrrr}
\hline Concepto & Año 0 & Año 1 & Año 2 & Año 3 & Año 4 & Año 5 \\
\hline Ingresos & - & 12,068 & 12,068 & 12,068 & 12,068 & 33,743 \\
Venta de pescado & & 12,068 & 12,068 & 12,068 & 12,068 & 12,068 \\
Valor residual & & & & & & 21,675 \\
Egresos & 28,205 & 7,268 & 7,268 & 7,268 & 7,268 & 7,268 \\
Inversiones & 28,205 & - & - & - & - & - \\
Inversión fija & 26,010 & & & & & \\
Equipos & 2,195 & & & & & \\
Costo de producción & & 7,268 & 7,268 & 7,268 & 7,268 & 7,268 \\
Personal & & 720 & 720 & 720 & 720 & 720 \\
Preparación de estanques & & 1,650 & 1,650 & 1,650 & 1,650 & 1,650 \\
Siembra & & 1,500 & 1,500 & 1,500 & 1,500 & 1,500 \\
Alimentación de peces & & 3,157 & 3,157 & 3,157 & 3,157 & 3,157 \\
Cosecha y comercialización & 241 & 241 & 241 & 241 & 241 \\
Saldo de caja & $(28,205)$ & 4,799 & 4,799 & 4,799 & 4,799 & 26,414 \\
\hline
\end{tabular}

Tabla 2. Flujo de caja para el tratamiento de 10000 peces $\mathrm{x} \mathrm{ha}^{-1}$

\begin{tabular}{|c|c|c|c|c|c|c|}
\hline Concepto & Año 0 & Año 1 & Año 2 & Año 3 & Año 4 & Año 5 \\
\hline Ingresos & - & 20,402 & 20,402 & 20,402 & 20,402 & 42,077 \\
\hline Venta de pescado & & 20,402 & 20,402 & 20,402 & 20,402 & 20,402 \\
\hline Valor residual & & & & & & 21,675 \\
\hline Egresos & 28,205 & 11,682 & 11,682 & 11,682 & 11,682 & 11,682 \\
\hline Inversiones & 28,205 & - & - & - & - & - \\
\hline Inversión fija & 26,010 & & & & & \\
\hline Equipos & 2,195 & & & & & \\
\hline Costo de producción & & 11,682 & 11,682 & 11,682 & 11,682 & 11,682 \\
\hline Personal & & 720 & 720 & 720 & 720 & 720 \\
\hline Preparación de estanques & & 1,650 & 1,650 & 1,650 & 1,650 & 1,650 \\
\hline Siembra & & 3000 & 3000 & 3000 & 3000 & 3000 \\
\hline Alimentación de peces & & 5,904 & 5,904 & 5,904 & 5,904 & 5,904 \\
\hline Cosecha y comercialización & & 408 & 408 & 408 & 408 & 408 \\
\hline Saldo de caja & $(28,205)$ & 8,720 & 8,720 & 8,720 & 8,720 & 30,395 \\
\hline
\end{tabular}


Tabla 3. Flujo de caja para el tratamiento de 15000 peces $\times$ ha $^{-1}$

\begin{tabular}{rrrrrrr}
\hline Concepto & Año 0 & Año 1 & Año 2 & Año 3 & Año 4 & Año 5 \\
\hline Ingresos & - & 27,962 & 27,962 & 27,962 & 27,962 & 49,637 \\
Venta de pescado & & 27,962 & 27,962 & 27,962 & 27,962 & 27,962 \\
Valor residual & & & & & & 21,675 \\
Egresos & 28,205 & 15,491 & 15,491 & 15,491 & 15,491 & 15,491 \\
Inversiones & 28,205 & - & - & - & - & - \\
Inversión fija & 26,010 & & & & & \\
Equipos & 2,195 & & & & & \\
Costo de producción & & 15,491 & 15,491 & 15,491 & 15,491 & 15,491 \\
Personal & & 720 & 720 & 720 & 720 & 720 \\
Preparación de estanques & & 1,650 & 1,650 & 1,650 & 1,650 & 1,650 \\
Siembra & & 4,500 & 4,500 & 4,500 & 4,500 & 4,500 \\
Alimentación de peces & & 8,061 & 8,061 & 8,061 & 8,061 & 8,061 \\
Cosecha y comercialización & 559 & 559 & 559 & 559 & 559 \\
Saldo de caja & $(28,205)$ & 12,471 & 12,471 & 12,471 & 12,471 & 34,146 \\
\hline
\end{tabular}

